\title{
Estimation of Contaminants for Direct Imaging of Exoplanets: Constraint on the Stellar Distribution Model with both NIR and Deep Imaging Data
}

\author{
Mihoko Konishi ${ }^{1}$, Hiroshi Shibai ${ }^{1}$, Taro Matsuo ${ }^{2}$, Kodai Yamamoto ${ }^{1}$, \\ Jun Sudo ${ }^{1}$, Matthias S. Samland ${ }^{1}$, Misato Fukagawa ${ }^{1}$, \\ Takahiro Sumi ${ }^{1}$ and SEEDS team \\ ${ }^{1}$ Department of Earth and Space Science, Graduate School of Science, Osaka University, \\ 1-1 Machikaneyama-cho, Toyonaka-shi, Osaka, 560-0043, Japan \\ email: konishi@iral.ess.sci.osaka-u.ac.jp \\ ${ }^{2}$ Department of Astronomy, Faculty of Science, Kyoto University, \\ Kitashirakawa-Oiwake-cho, Sakyo-ku, Kyoto, 606-8502, Japan
}

\begin{abstract}
There are faint contaminants near primary stars in the direct imaging of exoplanets. Our goal is to estimate statistically the ratio of exoplanets in the detected batch of point sources by calculating the fraction of contamination. In this study, we compared the detected number of stars with the number of contaminants predicted by our model. We found that the observed number of faint stars were fewer than the predicted results towards the Pleiades and GOODSSouth field when the parameters of the conventional stellar distribution models were employed. We thus estimated new model parameters in correspondence to the results of the observations.
\end{abstract}

Keywords. infrared: stars - Galaxy: stellar content - Galaxy: fundamental parameters - stars: low-mass, brown dwarfs - stars: luminosity function, mass function

\section{Motivation}

The goal of our study is to estimate statistically the ratio of exoplanets out of the detected number of point sources. In other words, we want to know strictly how many stars are contaminating the images. The number of contaminants can be found by using stellar distribution models of the Galaxy. Present stellar distribution models, however, are not constructed using both NIR and deep imaging data. So, we compared the observed number of stars to the number of stars predicted by the stellar distribution models of the Galaxy due to check whether they are consistent or not.

\section{Models}

For contaminants, we focused on late-type main-sequence field stars. We employed the stellar distribution models composed of a thin disk, thick disk, and halo to predict the number of contaminants for every sub-spectral type from K2 to T7. According to previous works (Gilmore \& Reid 1983; Gould et al. 1998; Cabalello et al. 2008; Chang et al. 2010; Chang et al. 2012),

Thin \& Thick Disk: $\mathcal{D}_{i, j}(R, Z)=n_{\odot, i} \times f_{d, j} \times \exp \left[-\frac{R-R_{\odot}}{h_{R, j}}-\frac{\left|Z-Z_{\odot}\right|}{h_{Z, j}}\right]$

$$
\text { Halo: } \mathcal{H}_{i}(R, Z)=n_{\odot, i} \times f_{h} \times\left[\frac{R^{2}+(Z / \kappa)^{2}}{R_{\odot}^{2}+\left(Z_{\odot} / \kappa\right)^{2}}\right]^{-\frac{p}{2}}
$$




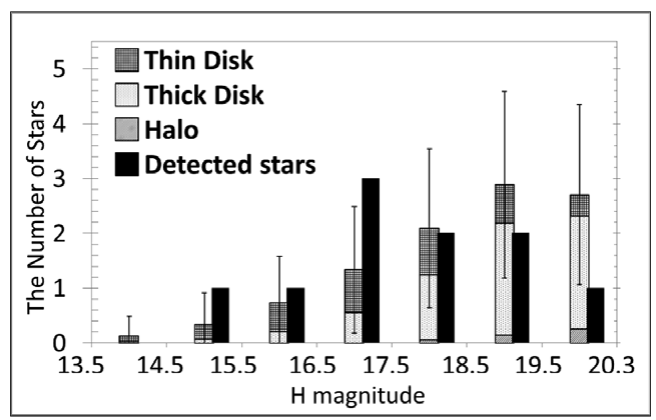

Figure 1. Observed and predicted numbers of contaminants towards the Pleiades open cluster

where $j$ indicates the thin or thick disk, $n_{\odot}$ is the local stellar density, $f_{d, j}$ and $f_{h}$ indicate the ratio of each component, $h_{R, j}$ and $h_{Z, j}$ indicate the scale height of the disk, $\kappa$ is the flattening parameter of the halo, $p$ is the power low index of the halo, and $\left(R_{\odot}, Z_{\odot}\right)$ is the Sun's position. For local stellar densities, we adopted values derived by the previous works (Cabalello et al. 2008; Reid et al. 2004; Reid et al. 2008).

\section{Data}

We used observational results of two areas; the Pleiades open cluster (Yamamoto et al. 2013) and the GOODS-South field (Windhorst et al. 2011). The Pleiades data were obtained with Subaru+HiCIAO/AO188. The FoV was $19^{\prime \prime} .5 \times 19^{\prime \prime} .5$. Twenty Pleiades member stars were observed. The median detection limit was $20.3 \mathrm{mag}$ in $H$-band. The GOODS-South field data were obtained with HST+WFC3. The FoV was $9^{\prime} .75 \times 4^{\prime} .5$. The detection limit with F160W filter was 25 mag.

\section{Results and Discussion}

Figure 1 shows the observed and predicted numbers of stars with increasing magnitude towards the Pleiades. We found that the detected number of faint stars was different from the number predicted from existing Galactic models. The predicted numbers of faint stars towards the Pleiades and the GOODS-South field were larger than observed numbers by two and about a few thousand, respectively. We thus adjusted parameters of the Galactic models so as to fit them to our observational results. Based on the results of the Pleiades observations, the scale height of the thick disk may be smaller than that previously thought. Also, from the observations of the GOODS-South field, the number of M-type stars in the halo was remarkably smaller than predicted from existing Galactic models.

The stellar distribution of M-type stars in the Galaxy is made clearer by both NIR and deep imaging.

\section{References}

Caballero, J. A., Burgasser, A. J., \& Klenment, R. 2008, A\&̈A, 488, 188

Chang, C.-K., Ko, C.-M., \& Peng, T.-H. 2010, ApJ, 724, 182

Chang, C.-K., Lai, S.-Y., Ko, C.-M., \& Peng, T.-H 2012, ApJ, 759, 94

Gilmore, G. \& Reid, N. 1983, MNRAS, 202, 1025

Gould, A., Flynn, C., \& Bahcall, J. N. 1998, ApJ, 503, 798

Reid, I. N., Cruz, K. L. et al. 2004, AJ, 128, 463

Reid, I. N., Cruz, K. L. et al. 2008, AJ, 136, 1290

Windhorst, R. A., Cohen, S. H. et al. 2011, ApJS, 193, 27

Yamamoto, K., Matsuo, T. et al. 2013, PASJ, 65, 90 